\title{
Prevalence of tobacco and alcohol consumption and associated factors in adults living with HIV infection
}

\author{
Rita Rocio Marquez Diaz \\ Hospital Clínico San Carlos, Madrid, Spain
}

\begin{abstract}
Introduction: Tobacco and alcohol consumption has a significant negative impact on health of individuals living with human immunodeficiency virus (HIV); however, there is little research on using these substances in this specific cohort. The objective of this study was to investigate the prevalence and factors associated with the consumption of tobacco and alcohol in adult population living with $\mathrm{HIV}$ as well as the clinical-epidemiological characteristics of the sample.

Material and methods: A cross-sectional study was carried out with 381 participants diagnosed with HIV, whose information on smoking and alcohol habits, adherence to treatments, socio-demographic data, and lifestyles were collected through both electronic and printed questionnaires.

Results: The results showed that half of the individuals were smokers, with significant percentage having a moderate or high motivation to quit smoking. Moreover, it was observed that the participants classified in the group of high alcohol consumption with AUDIT test were less adherent to pharmacological treatment. On the other hand, it should be noted that $49.3 \%$ of the sample suffered from some pathology, with cardiovascular disease being the most prevalent.

Conclusions: Therefore, from a clinical point of view and due to the above-mentioned reasons, it seems essential to address the consumption of tobacco and alcohol in individuals living with HIV. Along with proper health education and support offered, it can positively influence their lives in general, and at the same time, provide better HIV control.
\end{abstract}

HIV AIDS Rev 2021; 20, 3: 173-178 DOI: https://doi.org/10.5114/hivar.2021.108699

Key words: HIV, tobacco, alcohol, prevalence, surveys and questionnaires.

\section{Introduction}

Human immunodeficiency virus (HIV) is a disease that continues to be one of the biggest public health concerns. It is estimated that there are 36.7 million HIV-positive people living worldwide, and in Spain, infection rate is approximated to be 9.44 per 100,000 inhabitants, being among the 10 coun- tries in Europe with the highest rate of HIV diagnoses [1]. Since the first introduction of HIV-positive cases, there has been a remarkable evolution in terms of epidemiology and treatment of the disease [2]. Moreover, launching of highly active antiretroviral therapy (HAART) as therapy of choice in HIV infection, has reflected in a significant improvement in the quality and life expectancy of affected individuals [3].
Address for correspondence: Rita Rocio Marquez Diaz, Hospital Clínico San Carlos, Calle del Prof Martín Lagos, s/n, 28040 Madrid, Spain, e-mail: rociomard@hotmail.com
Article history:

Received: 21.03 .2021

Received in revised form: 26.04 .2021

Accepted: 27.04.2021

Available online: 30.08 .2021
International Journal of HIV-Related Problem

HIV \& AIDS Re vi e w 
However, benefits of treatment are closely related to strict compliance to HAART, and the consumption of certain substances, particularly alcohol, was considered an indicator factor in the lack of adherence of seropositive individuals [3]. Different studies had disagreed on whether alcohol contributes to an increase of toxicity in the liver due to HAART as well as an escalation of risk of HIV transmission in situations of alcohol intoxication and early cognitive deterioration [4]. Furthermore, harmful consumption of alcohol has been considered related to a worsening of immune state independently from the treatment [5]. For these reasons, it is important to thoroughly explore the matter of alcohol consumption while approaching HIV patients in order to intervene and seek better treatment of the disease [6].

In addition to harmful effects of alcohol consumption, it is necessary to highlight bad consequences of tobacco use on HIV individuals' health. Smoking has been identified as a significant co-factor in the premature development of cardiovascular disease related to HIV, increasing the risk of suffering an acute myocardial infarction or hypertension [7]. Moreover, smoking favors the increase of various diseases, including severe pulmonary infections, and a decrease in the quality of life of HIV-infected individuals [7]. According to recent studies, the risk of mortality is five times higher in seropositive smokers than in patients with HIV who have never smoked [8].

Because of these investigations, it seems important to recognize the prevalence of seropositive persons who are smokers, and to incorporate counseling and support programs into routine follow-up visits [9]. In this way, individuals can comprehend the risks of smoking in the context of HIV infection as well as to receive appropriate help in case they decide to quit smoking [10]. Finally, as per previous comparative studies, tobacco consumption in HIV population was shown higher than its prevalence in general population, ranging from $32 \%$ to $50 \%$ [11-16].

Despite the fact that smoking and alcohol consumption have a significant negative impact on health of individuals living with HIV, there is scarce research related to the use of this substances in HIV population [11, 15]. Having said this, the purpose of the present study was to investigate the current situation within this population and strategies that can be developed according to the results, and considering the importance of the involvement of health professionals in the process of smoking and alcohol cessation.

The objectives of this study were to assess the prevalence and factors associated with the consumption of tobacco and alcohol in adults living with HIV as well as clinicalepidemiological characteristics of the study sample.

\section{Material and methods}

A descriptive, cross-sectional analysis was performed. The study group consisted of adult individuals diagnosed with HIV, who attended infectious diseases consultation at the San Carlos Clinical Hospital, Madrid, Spain. A random sample of 369 individuals was calculated, accepting an alpha risk of 0.05 for a precision of \pm 0.05 percentage units, and based on data from previous studies, assuming an estimate of the proportion of population as $40 \%$.

With regard to measuring instruments, AUDIT [17] (alcohol use disorders identification test) and AUDIT-C [18] (abbreviated version of AUDIT) questionnaires were used to evaluate alcohol consumption. Additionally, hetero-administered test was applied with its items originated from global tobacco survey in adults (internationally known as "global adult tobacco survey" - GATS) to distinguish tobacco use. Smoking population within the study group carried out Richmond's questionnaire [19] on motivation to stop smoking. Furthermore, socio-demographic variables of the individuals were collected, including age, sex, nationality, marital status, occupation, practice of physical exercise as well as clinical characteristics, such as personal history, year of HIV diagnosis, and antiretroviral treatment, with its adherence measured using validated questionnaire SMAQ (simplified medication adherence questionnaire). The participants were classified according to year of HIV diagnosis, and divided into three groups: before the year 2000, between 2000 and 2010 , and from 2011 to 2019. All this information were collected in two different ways (online and in print) and were stored in an anonymized database, to which only the principal investigator was granted an access.

A descriptive analysis of the data was carried out evaluating mean standard deviation obtained from quantitative variables as well as mean $95 \%$ confidence interval. As for quantitative variables, which showed an asymmetric distribution, they were summarized by median and interquartile ranges. Qualitative variables were represented by the distribution of percentages of each category to estimate total prevalence.

To assess the association between quantitative variables, Pearson correlation coefficient was applied, whereas for categorical variables, $\chi^{2}$ test was used with Fisher's exact test or likelihood ratio, depending on the application conditions. To analyze the differences between two means, Student's $t$-test was applied for variables with normal distribution, and differences of means in more than two groups were analyzed using ANOVA test, for which assumptions of normality of the observations in different groups (KolmogorovSmirnoff), homoscedasticity of variances (Levene's test), and independence were fulfilled. Additionally, when homogeneity was met, Tukey test was applied for post-hoc tests.

Finally, in order to verify the hypothesis that the prevalence of smokers among individuals with HIV is higher than in the general population, $Z$-test was used for a binomial proportion. All analyses were executed with statistical program IBM SPSS version 21.0, and a $p$-value $<0.05$ was considered statistically significant.

This study was approved by ethical committee of the San Carlos Clinical Hospital of Madrid, Spain, and all the subjects participated voluntarily, with their informed consent provided. 


\section{Results}

\section{Socio-demographic and clinical characteristics of the study population}

In the present study, 381 subjects were included, with $88.9 \%$ of men and $11.1 \%$ of women. $57.2 \%$ of the total sample was between 35-54 years old, with an average age of $43.4 \pm 11.5$ years. 255 subjects completed the questionnaire electronically and 126 on paper. Regarding marital status, most men were single (76.1\%), while in in women, the majority were widowed, married, or divorced (59.5\%). Concerning subjects' employment position, $80 \%$ were active, $11.3 \%$ were unemployed, and $8.7 \%$ were retired. In the group of working participants, according to the National Classification of Economic Activities (CNAE), administrative and health activities were the most prevalent. Statistically significant differences were found between number of diseases and occupational status, showing those inactive having more illnesses than in other groups compared $(p<0.05)$. Concerning origin, $71.2 \%$ were Europeans, $20.7 \%$ were South Americans, and $8.1 \%$ originated from Central America, Caribbean, and North America regions.

The median of years since HIV diagnosis was 9 years (IQR, 11-15). Based on the results, there were statistically significant differences between years with HIV (calculated as the difference between 2019 and the year, in which an individual was diagnosed with the virus) and sex and smoking, showing that women and smokers had been diagnosed with HIV for more years $(p<0.05)$.

In this study, the participants were classified into three groups according to year of HIV diagnosis: before the year 2000, between 2000 and 2010 (both included), and from 2011 to present (2019), with $22 \%, 32 \%$, and $46 \%$ of participants, respectively. Besides HIV, 49.3\% subjects suffered from comorbid diseases, while $50.7 \%$ did not. Considering international classification of diseases (ICD-10), circulatory system disorders were most predominant, followed by mental and behavioral and digestive system diseases, particularly hypertension (HBP), depression, and hepatitis, respectively. In addition, it was found that there was a significant increase in the number of diseases with respect to sex and type of questionnaire performed. Subsequently, females, $\chi^{2}(1)=7.332, p=0.014$, and the participants who completed electronic questionnaires, $\chi^{2}(1)=7.030, p=0.019$, described additional number of diseases.

99.7\% of the study population was under ART, with Genvoya ${ }^{\circledR}$, Triumeq ${ }^{\circledR}$, and Truvada ${ }^{\circledR}$ as the most commonly drugs used. According to the score of SMAQ adhesion test, $52.8 \%$ of the subjects was adherent, while $47.2 \%$ was not. There was a significant reduction in adherence according to the age of an individual, showing younger subjects as less adherent $(p<0.05)$. Moreover, there was a statistically significant association between the type of questionnaire completed and adherence, indicating that those who performed electronic questionnaires were less adherent compared to those who completed questionnaires via paper, $\chi^{2}(1)=5.273, p=0.222$.
$57 \%$ of the subjects practiced physical exercise $>30$ minutes a day, therefore, they were considered active, while $43 \%$ of them were leading sedentary life-style. From here, it was possible to indicate a significant dependence between the practice of physical exercise and association of comorbid diseases, showing those who did not exercise having more diseases, $\chi^{2}(1)=4.349, p=0.037$. In addition, there was a dependency between physical exercise and being a smoker, demonstrating non-smokers tendency to be more active.

\section{Characteristics associated with smoking}

$49.1 \%$ of the participants were smokers, $27.8 \%$ ex-smokers, and $23.1 \%$ non-smokers. Table 1 shows main characteristics of the participants. Z-test for binomial proportion indicated that the prevalence of current smokers within the study group was significantly higher than that in the general population $(p<0.01)$. Those who smoke were inquired about their motivation to stop smoking, concluding that these individuals were very keen to quit smoking (seen question 2 of Richmond test [19]), but when trying to do so, they considered it doubtful (seen questions 3 and 4 of Richmond test [19]). Table 2 summarizes factors associated with tobacco consumption of participants who currently smoke.

According to different statistical results, it was assumed that variables, such as sex, occupation, origin, practice of physical exercise, awareness of the disease, and year of HIV diagnosis, differed significantly when compared to number of years of smoking, with $p<0.05$. Women, retired, Europeans, leading sedentary life-style, ill, and with HIV diagnosis prior to the year 2000, had smoked for more years. Similarly, a strong and positive correlation was observed between the number of years ofsmoking and Richmond test score $(r=0.869, p<0.05)$, showing that the more years the individual had been smoking, the bigger motivation to quit the habit.

Regarding the relationship between smoking and alcohol consumption, smokers showed a higher risk of consumption, as indicated by AUDIT questionnaire, $\chi^{2}(1)=5.279$, $p=0.022$.

\section{Consumption of alcohol}

Table 3 summarizes AUDIT and AUDIT-C tests scores of the study. The mean AUDIT test was $1.1 \pm 2.2$ (range, 0.89-1.34), while the mean AUDIT-C test was $2.8 \pm 2.3$ (range, 2.64-3.11).

AUDIT and AUDIT-C tests scores differed significantly according to age of participants, sex, occupational status, and year of HIV diagnosis, with a 95\% confidence $(p<0.05)$. At this point, it could be assumed that youngest male subjects, currently unemployed, and diagnosed between 2000 and 2010, presented a high alcohol risk consumption compared to the rest of the group. On the other hand, contingency tables showed a statistically significant dependency 
Table 1. Clinical-epidemiological characteristics of the study participants

\begin{tabular}{|c|c|c|c|c|}
\hline Variables & Former smoker & Smoker & Non-smoker & Total \\
\hline Age (years), mean \pm SD & $45.8 \pm 12.0$ & $43.5 \pm 10.6$ & $40.5 \pm 12.2$ & $43.4 \pm 11.5$ \\
\hline Years with HIV, Me; IQR & $10 ; 14$ & $9 ; 14$ & $6 ; 11$ & $9 ; 15$ \\
\hline \multicolumn{5}{|l|}{ Sex, $n(\%)$} \\
\hline Female & $9(8.5)$ & $25(13.4)$ & $8(9.1)$ & $42(11.0)$ \\
\hline Male & $97(91.5)$ & $162(86.6)$ & $80(90.9)$ & $339(89.0)$ \\
\hline \multicolumn{5}{|l|}{ Region, $n$ (\%) } \\
\hline South America & $27(25.5)$ & $29(15.5)$ & $23(26.1)$ & $79(20.7)$ \\
\hline Europe & $70(66.0)$ & $145(77.5)$ & $56(63.6)$ & $271(71.1)$ \\
\hline Other" & $9(8.5)$ & $13(7.0)$ & $9(10.2)$ & $31(8.1)$ \\
\hline \multicolumn{5}{|l|}{ Civil state, $n(\%)$} \\
\hline Married & $18(17.0)$ & $31(16.6)$ & $19(21.6)$ & $68(17.8)$ \\
\hline Divorced & $7(6.6)$ & $10(5.3)$ & $4(4.5)$ & $21(5.5)$ \\
\hline Separated & $1(0.9)$ & $7(3.7)$ & $0(0)$ & $8(2.1)$ \\
\hline Single & $74(69.8)$ & $135(72.2)$ & $64(72.7)$ & $273(71.7)$ \\
\hline Widowed & $6(5.7)$ & $4(2.1)$ & $1(1.1)$ & $11(2.9)$ \\
\hline \multicolumn{5}{|l|}{ Occupation, $n(\%)$} \\
\hline Active & $84(79.2)$ & $149(79.7)$ & $72(81.8)$ & $305(80.1)$ \\
\hline Unemployed & $11(10.4)$ & $21(11.2)$ & $11(12.5)$ & $43(11.3)$ \\
\hline Retired & $11(10.4)$ & $17(9.1)$ & $5(5.7)$ & $33(8.7)$ \\
\hline \multicolumn{5}{|l|}{ Diseases (ICD-10), $n(\%)$} \\
\hline With disease & $59(55.7)$ & $92(49.2)$ & $37(42.0)$ & $188(49.3)$ \\
\hline Without disease & $47(44.3)$ & $95(50.8)$ & $51(58.0)$ & $193(50.7)$ \\
\hline Cardiovascular disease, $n(\%)$ & $24(38.1)$ & $24(38.1)$ & $15(23.8)$ & $63(100.0)$ \\
\hline \multicolumn{5}{|l|}{ Physical exercise, $n(\%)$} \\
\hline Sedentary & $40(37.7)$ & $90(48.1)$ & $34(38.6)$ & $164(43.0)$ \\
\hline Active & $66(62.3)$ & 97 (51.9) & $54(61.4)$ & $217(57.0)$ \\
\hline
\end{tabular}

"Central America, Caribbean, and North America regions.

Table 2. Aspects associated with tobacco consumption of current smokers $(n=187)$

\begin{tabular}{|c|c|}
\hline Variables & \\
\hline \multicolumn{2}{|l|}{ No. of cigarettes per day, $n$ (\%) } \\
\hline$<10$ & $94(50.3)$ \\
\hline $10-20$ & $57(30.5)$ \\
\hline$>20$ & $36(19.3)$ \\
\hline \multicolumn{2}{|c|}{ RICHMOND test punctuation, $n(\%)$} \\
\hline High & $28(15.0)$ \\
\hline Moderate & $44(23.5)$ \\
\hline Doubtful & $65(34.8)$ \\
\hline Null or low & $50(26.7)$ \\
\hline Years of smoking, mean \pm SD & $23.1 \pm 12.5$ \\
\hline
\end{tabular}

Threshold points detected on association of level of motivation and cessation of smoking habit -0-3: null or low, 4-5: doubtful, 6-7: moderate, 8-10: high.
Table 3. Alcohol consumption pattern of the study participants

\begin{tabular}{|c|c|}
\hline Variables & \\
\hline \multicolumn{2}{|l|}{ AUDIT" test punctuation, $n(\%)$} \\
\hline Risk consumption & $8(2.1)$ \\
\hline Harmful consumption or dependence & $2(0.5)$ \\
\hline Risk-free & $371(97.4)$ \\
\hline \multicolumn{2}{|l|}{ AUDIT-C $C^{\# \# ~ t e s t ~ p u n c t u a t i o n, ~} n(\%)$} \\
\hline Low or moderate consumption & $244(64.0)$ \\
\hline Risk of consumption & $5(36.0)$ \\
\hline
\end{tabular}

"Men: total score 0-7 indicate no problems with alcohol consumption, 8-12: risk consumption, and 13-40: harmful consumption or dependence. Women: total score 0-5: no problems with alcohol consumption, 6-12: risk consumption, 13-40: harmful consumption or dependence.

\#\#Men: score equal to or greater than 4 indicate risk consumption, score < 4: low or moderate consumption. Women: score equal to or greater than 3 indicate risk consumption, score < 3: low or moderate consumption. 
between the adherence and results of AUDIT-C tests, concluding that non-adherents presented a higher risk of consumption, $\chi^{2}(1)=5.814, p=0.016$. Moreover, statistically significant dependency was also observed between the type of questionnaire (online vs. paper) and result of AUDIT-C test, indicating that those who completed online questionnaires presented a higher risk of alcohol consumption in AUDIT-C test, $\chi^{2}(1)=21.235, p<0.010$.

\section{Discussion}

The present study showed that the prevalence of tobacco use was generally higher in people living with HIV than in HIV-negative individuals, both men and women. In Spain, $22.7 \%$ of the adult population smoke, while in the study sample, the rate reached almost $50 \%$, which was 2.2 times higher than the national rate [20]. Various international studies with a diverse provenance have reported similar results $[11,16]$. For example, a survey conducted in the United States concluded that HIV-positive adults represented a higher percentage of smokers compared to general population, with $42.3 \%$ compared to $20.6 \%$, respectively [11]. This finding was also similar for European countries, such as France [12] or Denmark [13], in which tobacco consumption was higher among HIV-infected. In a study from Africa [14] comparing smoking habits of HIV individuals to those without HIV, it was reported that the proportion of smokers with HIV was greater than in HIV-negative population. In general, there is a high prevalence of tobacco consumption in HIV-positive people, which was reported in both Eastern and Western countries, with oscillating figures ranging from $32 \%$ to $70 \%$ [11-16]. The cause of such a wide range, especially lower prevalence, may be due to declining global trend of smoking in recent years and higher awareness of health risks associated. Meanwhile, the reasons for high prevalence could include low socio-economic and educationallevel, psychiatric comorbidities, simultaneous use of drugs and alcohol, and stress [9]. It was also observed that the proportion of smokers among men was moderately higher than among women [21], a fact that is in line with other studies conducted in China [15] or Vietnam [16]. The cause of this gap may be due, among others, to physical, psychological, and social dependence caused by tobacco [7]. In the present sample, the smoking rate was six times higher depending on the sex.

Consequently, future interventions are necessary to slow down this progress in both HIV-positive men and women. On the other hand, the results of Richmond [19] scale showed that a relatively important proportion present a moderate or high motivation to stop smoking, and this fact could be considered as favorable to intervention. To date, there are numerous studies that have examined attitudes of individuals and available interventions to quit smoking [10]. However, obstacles that hinder the implementation of these anti-smoking strategies exist; it was found that an individual is more likely to stop smoking if he/she is provided with an appropriate information and tools $[9,10]$.
The prevalence of alcohol consumption observed in this study is in line with the estimates of other surveys conducted among general population and patients living with HIV [5]. The reason for this low prevalence may be due to their awareness of being ill and need to follow healthy lifestyle recommendations for the control of HIV [22]. However, it should be noted that those classified as high-risk in AUDIT test were non-adherent to pharmacological treatment, and this association between alcohol use and lack of adherence was shown in other studies [3]. It is scientifically proven evidence that adherence to ART plays crucial role in the prevention of morbidity and mortality among HIV individuals [3]. Therefore, it is important to identify these patients and implement appropriate measures to reduce the impact of alcohol consumption on adherence to treatment [3].

The study also revealed a significant prevalence of hypertension, hypercholesterolemia, smoking, and obesity, which are the risk factors for cardiovascular diseases [8]. Numerous studies have shown that patients with HIV present a higher risk of cardiovascular complications [8]. Tobacco promotes premature development of cardiovascular diseases, and this risk is well-known to smoking population [22]. The main interventions are counselling services, and many cases require anti-smoking medications [22].

Finally, it is convenient to argue about the decision to divide the population into strata according to year of HIV diagnosis. According to the literature, patients who have been living with HIV for more than 20 years demonstrate different characteristics than those recently diagnosed with HIV [2].

\section{Limitations}

The observational design of the study prevented causal inferences. In fact, the generalization of the study results is limited and should be used with caution, when extrapolating them into other populations. Moreover, in order to reach the desired sample size in a short time, it was decided to use an electronic (online) questionnaire in addition to traditional paper form. This fact could influence complete validity of some of the answers as well as sincerity of the participants. There was additional finding of the study showing that most of the participants were working in the areas of administration and health. Those working in health areas could demonstrate lower rate of alcohol and tobacco consumption, when compared to other areas of working.

\section{Conclusions}

In the present study, about half of the participants were smokers, a co-factor that is observed in certain pathologies and as addition to mortality rate. However, a moderate proportion of individuals revealed a high motivation to quit smoking, especially those who smoked for a long time. In addition, it was possible to demonstrate that those non-adherent to ART were consuming alcohol continuously. Therefore, it is important for health professionals to guide 
HIV-positive patients to achieve better control of the disease, and to implement strategies to support both alcohol and smoking cessation.

\section{Conflict of interest}

The authors have no conflict of interest.

\section{References}

1. World Health Organization. VIH/SIDA; 2018. Available from: http:// www.who.int/mediacentre/factsheets/fs360/es/ (Accessed: 09.03.2019).

2. Barré-Sinoussi F, Ross AL, Delfraissy JF. Past, present and future: 30 years of HIV research. Nat Rev Microbiol 2013; 11: 277-283.

3. Parry CD, Morojele NK, Myers BJ, Kekwaletswe CT, Manda SO, Sorsdahl K, et al. Efficacy of an alcohol-focused intervention for improving adherence to antiretroviral therapy (ART) and HIV treatment outcomes - a randomised controlled trial protocol. BMC Infect Dis 2014; 14: 500 .

4. Baliunas D, Rehm J, Irving H, Shuper P. Alcohol consumption and risk of incident human immunodeficiency virus infection: a metaanalysis. Int J Public Health 2010; 55: 159-166.

5. Kelso-Chichetto NE, Plankey M, Abraham AG, Ennis N, Chen X, Bolan R, et al. Association between alcohol consumption trajectories and clinical profiles among women and men living with HIV. Am J Drug Alcohol Abuse 2018; 44: 85-94

6. Elliott JC, Aharonovich E, Hasin DS. Reasons for limiting drinking in an HIV primary care sample. Alcohol Clin Exp Res 2014; 38: 1720-1727.

7. Calvo M, Laguno M, Martinez M, Martínez E. Effects of tobacco smoking on HIV-infected individuals. AIDS Rev 2014; 17: 47-55.

8. Calvo M, Martínez E. Riesgo cardiovascular e infección por el virus de la inmunodeficiencia humana. Enferm Infecc Microbiol Clin 2012; 30: 515-610.

9. Debra C, Michael BS, Polly T, Shobha S, Sally LH. Evaluation of a smoking cessation program for HIV infected individuals in an urban HIV clinic: challenges and lessons learned. AIDS Res Treat 2014; 2014: 237834.

10. Weinberger AH, Seng EK, Esan H, Shuter J. Perceived risks and benefits of quitting smoking in a sample of adults living with HIV/ AIDS. AIDS Care 2018; 30: 564-568.

11. Mdodo R, Frazier EL, Dube SR, Mattson CL, Sutton MY, Brooks JT, et al. Cigarette smoking prevalence among adults with HIV compared with the general adult population in the United States: cross-sectional surveys. Ann Intern Med 2015; 162: 335-344.

12. Tron L, Lert F, Spire B, Dray-Spira R; ANRS-Vespa2 study group. Tobacco smoking in HIV-infected versus general population in France: heterogeneity across the various groups of people living with HIV. PLoS One 2014; 9: e107451.

13. Helleberg M, Afzal S, Kronborg G, Larsen CS, Pedersen G, Pedersen C, et al. Mortality attributable to smoking among HIV-1-infected individuals: a nationwide, population-based cohort study. Clin Infect Dis 2013; 56: 727-734.

14. Mdege ND, Shah S, Ayo-Yusuf OA, Hakim J, Siddiqi K. Tobacco use among people living with HIV: analysis of data from demographic and Health Surveys from 28 low-income and middle-income countries. Lancet Glob Health 2017; 5: e578-e592.

15. Jin ZY, Liu X, Ding YY, Zhang ZF, He N. Cancer risk factors among people living with HIV/AIDS in China: a systematic review and meta-analysis. Sci Rep 2017; 7: 4890.

16. Nguyen NP, Tran BX, Hwang LY, Markham CM, Swartz MD, Phan HTT, et al. Prevalence of cigarette smoking and associated factors in a large sample of HIV-positive patients receiving antiretroviral therapy in Vietnam. PLoS One 2015; 10: e0118185.
17. Saunders JB, Aasland OG, Babor TF, de la Fuente JR, Grant M. Development of the Alcohol Use Disorders Identification Test (AUDIT): WHO collaborative project on early detection of persons with harmful alcohol consumption-II. Addiction 1993; 88: 791-804.

18. Bush K, Kivlahan DR, McDonell MB, Fihn SD, Bradley KA. The AUDIT Alcohol Consumption Questions (AUDIT-C): an effective brief screening test for problem drinking. Arch Intern Med 1998; 158: 1789-1795.

19. Richmond RL, Kehoe LA, Webster IW. Multivariate models for predicting abstention following. Addiction 1993; 88: 1127-1135.

20. INE2019. Encuesta nacional de salud 2017. Madrid: Instituto Nacional de Estadística [Online]. Available from: http://www. ine.es/dyngs/INEbase/es/operacion.htm? c=Estadistica_C\&cid= $1254736176783 \&$ menu $=$ resultados $\& \sec c=1254736195650 \& \mathrm{ridp}=$ 1254735573175.

21. Shuter J, Pearlman BK, Stanton CA, Moadel AB, Kim RS, Weinberger AH. Gender differences among smokers living with HIV. J Int Assoc Provid AIDS Care 2016; 15: 412-417.

22. Brown JL, DeMartini KS, Sales JM, Swartzendruber AL, DiClemente RJ. Interventions to reduce alcohol use among HIV-infected individuals: a review and critique of the literature. Curr HIV/AIDS Rep 2013; 10: 356-370. 